\title{
Consumo de bebidas alcoólicas e prática do binge drinking entre acadêmicos de Odontologia de uma IES
}

\author{
Alcohol consumption and binge drinking among Dental students of a university
}

Simony dos Santos Saraiva

Graduanda em Odontologia da Faculdade Integral Diferencial (FACID/DeVry)

Antônio Luiz Martins Maia Filho

Doutor em Engenharia Biomédica pela Univap

Professor de Fisiologia da Universidade Estadual do Piauí (UESPI)

\section{Resumo}

0 objetivo deste estudo foi identificar a prevalência do consumo de álcool entre os acadêmicos de Odontologia e fatores associados ao binge drinking. Tratou-se de um estudo transversal, com amostra representativa, realizado numa faculdade privada de Teresina - PI. Utilizou-se um questionário estruturado com questões validadas para autopreenchimento. A associação entre variáveis foi aferida pelo teste qui-quadrado, assumindo-se o nivel de significância de $5 \%(p<0,05)$. Foram analisados 255 questionários. A prevalência do consumo de bebidas alcoólicas entre o grupo estudado foi de $50,2 \%$, e a prática do binge drinking apresentou prevalência de $18,4 \%$. Estes índices são significativamente altos e demandam estratégias específicas dos gestores e das instituições de ensino para o controle do problema.

Palavras-Chave: consumo de bebidas alcoólicas; estudantes de Odontologia; epidemiologia.

\section{Abstract}

The objective of this study was to identify the prevalence of alcohol consumption among dental college students and factors associated with binge drinking. This was a cross-sectional study with a representative sample, held on a private university Teresina - PI. A structured questionnaire with questions validated for self-assessment. The association between variables was assessed using the chi-square test, assuming a significance level of $5 \%(p<0.05)$. Two hundred fifty-five questionnaires were analyzed. The prevalence of alcohol consumption among the sample of the study was $50.2 \%$, and the once of binge drinking had a prevalence of $18.4 \%$. The difference of sex and villas presented with high significance ( $p$ $<0.01$ ). These rates are significantly high and require specific strategies from managers and educational institutions to get this problem under control. Keywords: alcohol drinking; dental students; epidemiology.

\section{Introdução}

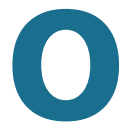
consumo de bebidas alcoólicas em grandes quantidades contribui na etiologia e manutenção de vários problemas sociais, econômicos e de saúde registrados no Brasil, e alguns estudos mostram que o seu uso tem início cada vez mais cedo (1).

Os jovens universitários são considerados a população mais vulnerável ao consumo de bebidas alcoólicas, esta é uma das maiores preocupações referentes à saúde e ao comportamento do estudante (2).

O consumo de álcool pode interferir no desempenho acadêmico dos estudantes devido às faltas ou ao baixo rendimento nas aulas, expor os jovens a problemas como acidentes de trânsito, comportamento sexual de risco, gravidez não planejada, violência, ferimentos não intencionais, entre outros (3).

A prática do binge drinking, que pode ser traduzido como 'beber episódico pesado', é empregado para definir o uso excessivo episódico do álcool quando a pessoa consome doses elevadas de bebidas alcoólicas em uma única ocasião (4).

Binge drinking significa beber em grandes quantidades até que os níveis de concentração de álcool no sangue possam atingir $0,08 \mathrm{~g} / \mathrm{dL}$. Para o adulto esse padrão é equivalente a cinco doses ou mais para homens e quatro ou mais para mulheres, consumido dentro de um prazo de 2 horas (5). Uma dose padrão de álcool contém 10-12 gramas de etanol puro, o binge drinking é a ingestão de seis doses em uma única ocasião o que corresponde a seis ou mais latas de cerveja (350 ml) ou seis doses de destilados ou seis taças de vinho (2).

Definições sobre o conceito de binge baseiam-se na quantidade de álcool consumida dentro de um período definido (ex.: um dia, uma ocasião, número de horas) e na frequência de consumo (ex.: semanalmente ou mensalmente) (6).

$\mathrm{O}$ presente estudo justifica-se por se tratar de uma questão relevante e um alerta aos gestores de saúde, além disso, existem poucos trabalhos que relacionam o consumo de álcool entre os jovens e seus efeitos no desempenho acadêmico (7). Em pesquisas nacionais, observa-se que diferentes metodologias são empregadas, dificultando assim a comparação dos dados (1).

O objetivo deste trabalho foi verificar a prevalência do consumo de bebidas alcoólicas entre acadêmicos de Odontologia, como também a prevalência e fatores associados ao binge drinking para mesma população, em uma instituição particular de ensino superior situado na cidade de Teresina - PI.

\section{Material e Método}

Trata-se de uma pesquisa transversal, exploratória e analítica, com uma abordagem quantitativa. Aprovado com onúmero CAEE32926014.9.0000.5211 pelo Comitê de Ética em Pesquisa (CEP) da Faculdade Integral Diferencial (FACID - DeVry). A pesquisa segue as normas do Conselho Nacional de Saúde, conforme a resolução 466/12.

A coleta de dados foi realizada em uma Faculdade Privada, na cidade de Teresina - PI. A amostra da pesquisa foi constituída pelos acadêmicos de Odontologia regularmente matriculados no ano de 2014, que estavam presentes nas salas de aula na data de aplicação do questionário e que aceitaram participar do projeto. Para a alocação dos respondentes, efetuou-se uma 
amostragem incluindo todos os alunos do primeiro ao décimo período de Odontologia.

O questionário aplicado foi constituído de perguntas fechadas com alternativas de respostas em categorias fixas e preestabelecidas. Esse instrumento de coleta de dados foi baseado no AUDIT (Alcohol Use Disorders Identification Test), questionário desenvolvido pela Organização Mundial de Saúde (OMS), como instrumento de rastreamento para classificar o consumo de bebidas alcoólicas (8). Também foram utilizadas questões validadas em outros estudos sobre o mesmo tema (9) e questões contemplando o perfil sociodemográfico do respondente, além da identificação dos problemas mais comuns vivenciados pelos acadêmicos, após o consumo de bebidas alcoólicas. Apesar de o AUDIT ser universalmente aceito e aplicado, não permite detalhamento sobre a quantidade de bebida alcoólica ingerida, segundo as diversas bebidas (destiladas ou fermentadas), e não alcança a precisão desejada, quando a coleta dos dados é realizada por questionário autoaplicativo. Após a coletada das informações, os entrevistados serão classificados em usuários ou não e como praticantes ou não do binge drinking.

Uma caixa simulando uma urna foi colocada sobre a mesa da sala de aula para que os questionários fossem colocados após seu preenchimento, a fim de que os acadêmicos não pudessem ser identificados.

Para a análise dos dados, foi utilizado o software SPSS, versão 17.0, assumindo-se o nível de significância de $5 \%$ (p < $0,05)$ na análise do teste estatístico utilizado (qui-quadrado).

\section{Resultados}

A pesquisa obteve a participação de 255 universitários do curso de Odontologia. A maior parte dos estudantes que aceitaram participar do estudo $(61,6 \%)$ cursava até o quinto período.

Registrou-se predomínio do sexo feminino $(67,45 \%)$ e da faixa etária entre 18 e 25 anos (87,84\%). A religião predominante foi a católica $(77,25 \%)$ e a maior parte dos estudantes referiu morar com a família $(63,92 \%)$.

No presente estudo, a bebida mais comumente utilizada foi cerveja. Ainda, entre os que fazem uso de álcool com maior frequência, ela também foi a bebida mais consumida, seguido dos destilados e do vinho (Tabela III).

A prevalência de uso ou consumo regular de bebidas alcoólicas entre os estudantes, independentemente da quantidade, foi de $50,2 \%(n=128)$. A bebida mais comumente utilizada foi a cerveja. Ainda, entre os que fazem uso de álcool com maior frequência, ela também foi a bebida mais consumida, seguido dos destilados e do vinho (Tabela I). A prevalência de casos de binge drinkig entre eles foi de $18,4 \%$ $(\mathrm{n}=47)$. Comparando-se a prevalência de binge em relação ao sexo, o masculino apresentou um percentual de $12,9 \%$ (n = 33) e o sexo feminino 5,5\% ( $\mathrm{n}=14)$ (Tabela II). Na tabela II, verificou-se que há diferença altamente significativa entre os acadêmicos de diferentes sexos e moradias $(\mathrm{P}<0,01)$.
Nesta pesquisa buscou-se identificar a ocorrência de eventos indesejáveis associados ou situações de risco, após o consumo de bebidas alcoólicas entre os universitários estudados. Destaca-se que mais de $40 \%$ dos estudantes declararam já ter dirigido após consumir bebidas alcoólicas e $18 \%$ já se envolveram em acidentes de trânsito. Neste estudo o sexo feminino (21\%) dirigiu mais vezes após consumir álcool do que o sexo masculino (19\%).

Um percentual elevado de estudantes, aproximadamente $32,5 \%$, não compareceu às atividades da universidade devido ao consumo de abusivo de bebidas alcoólicas, sendo que cerca de $30 \%$ apresentou um baixo desempenho em avaliações. Uma percentagem não desprezível de estudantes envolveu-se em brigas ou problemas com a lei $(7,4 \%)$, pelo uso de bebidas alcoólicas.

A tabela III revelou que para população estudada a prática do binge drinking esteve estatisticamente relacionada a práticas perigosas, como dirigir após consumo de álcool e desfechos indesejáveis, como envolvimento em acidentes de trânsito, brigas e problemas com a lei.

Tabela I. Principais tipos e frequências de bebidas consumidas por estudantes do curso de Odontologia de uma universidade particular (Teresina/PI - 2015)

\begin{tabular}{|c|c|c|}
\hline Variáveis & (n) & $(\%)$ \\
\hline \multicolumn{3}{|l|}{ Consumo de } \\
\hline \multicolumn{3}{|l|}{ Cerveja } \\
\hline $\begin{array}{l}<1 \text { vez por } \\
\text { semana }\end{array}$ & 100 & 39,22 \\
\hline $\begin{array}{l}1 \text { a } 4 \text { vezes por } \\
\text { semana }\end{array}$ & 27 & 10,59 \\
\hline $\begin{array}{l}\geq 5 \text { vezes por } \\
\text { semana }\end{array}$ & 1 & 0,39 \\
\hline Não bebe & 127 & 49,80 \\
\hline \multicolumn{3}{|l|}{ Consumo de } \\
\hline $\begin{array}{l}<1 \text { vez por } \\
\text { semana }\end{array}$ & 42 & 16,47 \\
\hline $\begin{array}{l}1 \text { a } 4 \text { vezes por } \\
\text { semana }\end{array}$ & 1 & 0,39 \\
\hline $\begin{array}{l}\geq 5 \text { vezes por } \\
\text { semana }\end{array}$ & 1 & 0,39 \\
\hline Năo bebe & 211 & 82,75 \\
\hline \multicolumn{3}{|l|}{ Consumo de } \\
\hline $\begin{array}{l}<1 \text { vez por } \\
\text { semana }\end{array}$ & 97 & 38,04 \\
\hline $\begin{array}{l}1 \text { a } 4 \text { vezes por } \\
\text { semana }\end{array}$ & 16 & 6,27 \\
\hline $\begin{array}{l}\geq 5 \text { vezes por } \\
\text { semana }\end{array}$ & 1. & 0,39 \\
\hline Não bebe & 141 & 55,30 \\
\hline
\end{tabular}

Fonte: SARAIVA (2015) 
Tabela II. Associação entre variáveis estudadas e a prática do binge drinking entre os estudantes do curso de Odontologia de uma universidade particular (Teresina/PI - 2015)

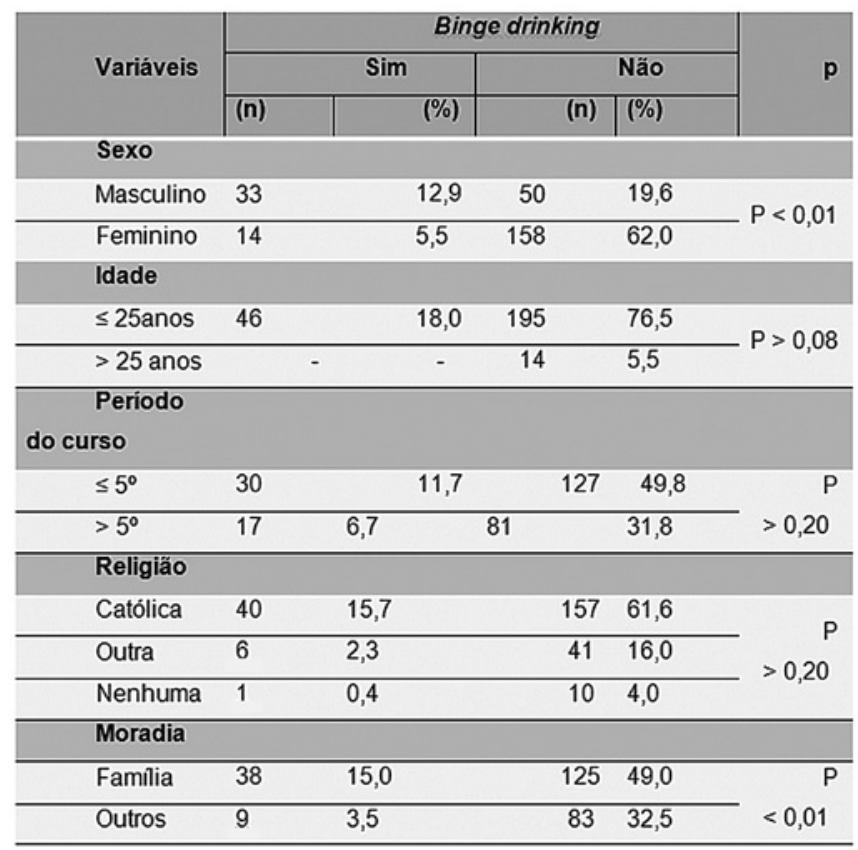

\section{Discussão}

O consumo de bebidas alcoólicas de forma abusiva implica em riscos tanto para quem consome como para as pessoas em seu entorno. Para a amostra da população estudada, a prevalência do consumo de bebidas alcoólicas em geral foi consideravelmente alta. Os resultados são concordantes com outros estudos que também apresentam frequências elevadas do consumo de álcool. Um estudo similar realizado, em Minas Gerais, com acadêmicos na área de saúde, revelou uma prevalência $71,5 \%$ para o consumo de álcool (4). O I Levantamento Nacional sobre o Uso de Álcool, Tabaco e outras Drogas entre Universitários (10), realizado com amostras de estudantes de 27 capitais brasileiras, evidenciou a prevalência de consumo de bebidas alcoólicas para os últimos 12 meses de $72 \%$.

Muitos estudos sobre o consumo de bebidas alcoólicas não mencionam o tipo de bebida mais consumida entre os jovens. Neste estudo a cerveja apresentou uma maior frequência de consumo. Esse dado corrobora com os resultados do Relatório Global sobre o Álcool e Saúde (11), na qual a cerveja (55\%) é o tipo mais consumido na Região das Américas, seguido dos destilados $(32,6 \%)$ e do vinho $(11,7 \%)$. A cerveja foi apontada como a bebida mais consumida por terem sido anunciadas nas mídias (televisão, jornais e/ou revistas) (12).

Segundo pesquisa realizada com alunos de Medicina de uma Faculdade de Minas Gerais, 54,9\% desses estudantes consumiram até seis doses em um dia típico e $8,8 \%$ acima de sete doses (13). No II Levantamento Nacional de Álcool e Drogas (14), 39\% dos entrevistados relataram que consomem cinco doses ou mais de bebidas alcoólicas em uma úni-
Tabela III. Associação entre situações de risco ou eventos indesejáveis e a prática do binge drinking entre os estudantes do curso de Odontologia de uma universidade particular (Teresina/PI - 2015) (Teresina/PI - 2015)

\begin{tabular}{|c|c|c|c|c|c|c|}
\hline \multirow{3}{*}{ Variáveis } & \multicolumn{4}{|c|}{ Binge drinking } & & \multirow{3}{*}{ p } \\
\hline & \multicolumn{2}{|r|}{ Sim } & \multicolumn{3}{|c|}{ Näo } & \\
\hline & n) & $(\%)$ & (n) & $(\%)$ & & \\
\hline \multicolumn{7}{|c|}{ Dirigir após beber } \\
\hline Sim & 8 & 14,9 & 65 & 25,5 & & \multirow{2}{*}{$\begin{array}{l}p< \\
0,01\end{array}$} \\
\hline Não & 6 & 6,3 & 136 & 53,3 & & \\
\hline \multicolumn{7}{|c|}{ Envolvimento em acidentes de trânsito } \\
\hline Sim & 5 & 5,9 & 31 & 12,2 & & \multirow{2}{*}{$\begin{array}{l}p> \\
0,06\end{array}$} \\
\hline Não & 3 & 12,9 & 176 & 69,0 & & \\
\hline \multicolumn{7}{|c|}{ Não compareceu à faculdade } \\
\hline Sim & 3 & 12,9 & 50 & 19,6 & & \multirow{2}{*}{$\begin{array}{l}p< \\
0,01\end{array}$} \\
\hline Não & 4 & 5,5 & 158 & 62,0 & & \\
\hline \multicolumn{7}{|c|}{ Baixo desempenho em avaliaçöes } \\
\hline Sim & 3 & 12,9 & 41 & 16,1 & & \multirow{2}{*}{$\begin{array}{r}p \\
<0,01\end{array}$} \\
\hline Não & 5 & 5,9 & 166 & 65,1 & & \\
\hline \multicolumn{7}{|c|}{ Envolver-se em brigas ou problemas com lei } \\
\hline Sim & 2 & 4,70 & 7 & & 2,8 & \multirow{2}{*}{$\begin{array}{r}p \\
<0,01\end{array}$} \\
\hline Não & 4 & 13,3 & 202 & 79,2 & & \\
\hline
\end{tabular}

Fonte: SARAIVA (2015)

ca ocasião. No presente estudo não foi possível quantificar as doses consumidas pelos estudantes, pois no questionário aplicado não fazia referências a quantidades, apenas a frequências. O comportamento de beber muitas doses em uma única ocasião está mais presente na faixa de 18 a 24 anos (15). Nesta pesquisa observou-se que, da população estuda$\mathrm{da}$ ( $\mathrm{n}=255$ estudantes), $87,8 \%$ estavam na faixa entre $18 \mathrm{e}$ 25 anos.

Estudo realizado em todo território brasileiro mostrou que $40 \%$ dos jovens adultos entrevistados relataram pelo menos uma ocasião de binge drinking nos últimos 12 meses (1). O I Levantamento Nacional sobre o Uso de Álcool, Tabaco e outras Drogas entre Universitários (10) evidenciou uma prevalência de consumo em binge, nos últimos 12 meses de $35,7 \%$, essa prática ainda foi maior entre os homens $(43,7 \%)$ do que entre as mulheres $(29 \%)$. A prevalência de ocorrência do binge drinking entre os estudantes pesquisados foi inferior aos índices nacionais. Essa diferença pode ter ocorrido pelo fato de a maior parte dos estudantes pesquisados neste estudo pertencerem ao sexo feminino. Os homens consomem bebida alcoólica numa frequência de 3,6 
vezes por semana, enquanto que as mulheres consomem 1,9 vezes por semana (9).

Os problemas provocados pelas bebidas alcoólicas variam desde acidentes de trânsito, comportamento sexual de risco (doenças sexualmente transmissíveis, gravidez indesejada), violência, ferimentos não intencionais e problema acadêmicos (16).

Em Pesquisa Nacional de Saúde (17), o percentual de indivíduos que conduziram veículo motorizado (carro ou motocicleta) após o consumo de álcool independente da quantidade e da periodicidade da prática foi de $24,3 \%$. Ainda, quando comparado entre os sexos, os homens (27,4\%) dirigiriam mais vezes após o consumo de álcool do que as mulheres (11,9\%). Esses dados divergem do encontrado neste estudo, na qual evidenciou que o sexo feminino (21\%) dirigiu mais vezes após consumir álcool do que o sexo masculino (19\%). Porém, essa diferença pode ter ocorrido por ter uma maior participação de mulheres neste estudo.

Sobre situações de risco ou eventos indesejáveis associados ao consumo de álcool, um estudo realizado com 474 estudantes da área da saúde revelou que mais de $20 \%$ dos alunos declararam já ter dirigido após o consumo de bebida alcoólico e 8 $\%$ já se envolveram em brigas ou problemas com a lei. Um percentual não desprezível de estudantes deixou de comparecer às atividades na universidade por conta da bebedeira (4). Esses dados assemelham-se aos encontrados nesta pesquisa, pois estes eventos também ocorreram na IES pesquisada, porém não apresentou porcentagens semelhantes.

Estudos comprovam que, embora a maioria dos jovens conheçam os riscos do uso abusivo do álcool, a busca por substâncias capazes de aliviar situações de estresse e ansiogênico resultaria no consumo em grandes quantidades de bebidas alcoólicas como um refúgio ocasional, mas que poderia chegar a consequências danosas $(13,18)$.

\section{Conclusão}

Verificou-se que existe uma alta prevalência de consumo de bebidas alcoólicas e de prática de binge drinking entre os acadêmicos de Odontologia podendo, desta forma, expor estes jovens a eventos indesejáveis ou situações de risco. Assim, fica evidente a importância da inclusão dos conteúdos sobre álcool nos currículos de graduação.

O tema abordado é amplo, portanto, sugere-se que novas pesquisas sejam desenvolvidas para que as Instituições de Ensino Superior e Gestores possam desenvolver programas específicos de intervenção e prevenção de consumo nocivo de bebidas alcoólicas entre os universitários. 


\section{Referências ::}

1. LARANJEIRA, R, et al. Alcohol use patterns among Brazilian adults. Rev Bras Psiquiatr 2010;32(3):231-41.

2. PILLON, SC, CORRADI-WEBSTER, CM. Teste de identificação de problemas relacionados ao uso de álcool entre universitários. Rev Enferm UERJ. 2006;14(3): 325-32.

3. BAUMGARTEN, LZ, FONSECA, AD, GOMES, VLO. Consumo alcoólico entre universitários (as) da área de saúde da Universidade Federal do Rio Grande/RS: subsídios para enfermagem. Esc Anna Nery 2012;16(3): 530-35.

4. NUNES, JM, et al. Consumo de bebidas alcoólicas e prática do binge drinking entre acadêmicos da área da saúde. Rev Psiq Clín 2012;39(3):94-9.

5. [NIAAA] National Institute on Alcohol and Alcoholism. Drinking levels defined. 2014. [Acesso em: 23 mai. 2014]. Disponível em: http://www. niaaa.nih.gov/alcohol-health/overview-alcohol-consumption/moderate-binge-drinking

6. PEUKER, AC, FOGAÇA, J, BIZARRO, L. Expectativas e beber problemático entre universitários. Psic Teor Pesq 2006; 22(2):193-200.

7. HOWLAND, J, et al. The Effects of binge drinking on college students' Next-Day Academic Test-Taking Performance and Mood State. NIH Public Access Author Manuscript. 2010, 105(4): 655-65.

8. MÉNDEZ, EB. Uma versão brasileira do AUDIT (Alcohol Use Disorders Identification Test) [dissertação]. Pelotas: Universidade Federal de Pelotas; 1999. 121f.

9. RIBEIRO, E. Padrão de consumo de bebidas alcoólicas entre universitários da área da saúde de uma faculdade do interior do estado de São Paulo [dissertação]. Ribeirão Preto: Escola de Enfermagem de Ribeirão Preto, Universidade de São Paulo; 2007. 125f.
10. ANDRADE, AG, DUARTE, PCAV, OLIVEIRA, LG. I Levantamento Nacional sobre o Uso de Álcool, Tabaco e Outras Drogas entre Universitários das 27 Capitais Brasileiras. Secretaria Nacional de Políticas sobre Drogas. Brasília: SENAD; 2010.

11. [WHO] World Health Organization. Global status report on alcohol and health. World Health Organization; Geneva. 2014.

12. PEDROSA, AAS, et al. Consumo de álcool entre estudantes universitários. Cad Saúde Pública 2011;27(8):1611-21.

13. ROCHA, LA, et al. Consumo de álcool entre estudantes de faculdades de medicina de Minas Gerais, Brasil. Rev. Bras. Educ. Méd. 2011;35(3):369-75.

14. LARANJEIRA, R, et al. II levantamento nacional de álcool e drogas (LENAD). Instituto Nacional de Ciência e Tecnologia para Políticas Públicas de álcool e outras drogas (INPAD). São Paulo: UNIFESP; 2014.

15. LARANJEIRA, R, et al. Levantamento Nacional sobre Padrões do Consumo de Álcool na População Brasileira. Brasília: Secretaria Nacional Antidrogas; 2007.

16. GOMES, BMR, ALVES, JGB, NASCIMENTO, LC. Consumo de álcool entre estudantes de escolas públicas da região metropolitana do Recife, Pernambuco, Brasil. Cad Saúde Pública 2010;26(4):706-12.

17. [IBGE] INSTITUTO BRASILEIRO DE GEOGRAFIA E ESTATÍSTICA. Pesquisa nacional de saúde 2013. Percepção do estado de saúde, estilos de vida e doenças crônicas: Brasil, grandes regiões e unidades da federação. Rio de Janeiro: IBGE; 2014.

18. PEUKER, AC, FOGAÇA, J, BIZARRO, L. Expectativas e beber problemático entre universitários. Psic Teor Pesq 2006;22(2):193-200.

Recebido em: 04/08/2014 / Aprovado em: 05/09/2014

Simony dos Santos Saraiva

Rua Tancredo Serra e Silva, 2049, Condomínio Sete Cidades, bloco 5/apto. 203 - Horto Florestal

Teresina/PI, Brasil - CEP: 64052-475

E-mail: symonysarayva@hotmail.com 